\title{
Research Paper \\ Comparing the Postoperative Sore Throat Incidence and Hemodynamic Changes after Using Macintosh and GlideScope Laryngoscopes and Laryngeal Mask Airway
}

\author{
Mohammad Ali Masoumifar ${ }^{1}$, Mahdi Ebtehaj ${ }^{1}$ (1), *Hamid Kayalha $^{1}$ (1), Ali Akbar Shafikhani ${ }^{2}$ (1)
}

1. Department of Anesthesiology, Metabolic Disease Research Center, Qazvin University of Medical Sciences, Qazvin, Iran.

2. Department of Occupational Health Engineering, School of Public Health, Shahid Beheshti University of Medical Sciences, Tehran, Iran.

\begin{tabular}{|l|l|l|l}
\hline $\begin{array}{l}\text { Use your device to scan } \\
\text { and read the article online }\end{array}$ & $\begin{array}{l}\text { Citation Masoumifar MA, Ebtehaj M, Kayalha H, Shafikhani AA. Comparing the Postoperative Sore Throat Incidence and Hemody- } \\
\text { namic Changes after Using Macintosh and GlideScope Laryngoscopes and Laryngeal Mask Airway. The Journal of Qazvin University of } \\
\text { Medical Sciences. 2020; 23(6):540-549. https://doi.org/10.32598/JQUMS.23.6.6 }\end{array}$ \\
dol'https://doi.org/10.32598/JQUMS.23.6.6
\end{tabular}

Received: 18 Sep 2019 Accepted: 23 Dec 2019 Available Online: 01 Feb 2020

Keywords: Anesthesiology, Laryngoscopy, Laryngeal Mask Airway

\section{A B STRACT}

Background Studies have shown that postoperative sore throat (POST) is the most common complication of tracheal intubation; however, its actual incidence and extent are not well documented.

Objective The aim of this study was to evaluate and compare the incidence of POST and hemodynamic changes after using macintosh laryngoscope (MCL), glidescope laryngoscope (GSL), and laryngeal mask airway (LMA) for elective surgery.

Methods This randomized double-blind clinical trial was conducted on 90 patients undergoing elective surgery in Shahid Rajaee Hospital in Qazvin, Iran. Patients were randomly divided into three groups based on the used intubation techniques including MCL, GSL, and LMA. The incidence of POST, hemodynamic changes, laryngoscopy duration, intubation duration, and number of attempts were compared in three groups and analyzed by ANOVA, independent t-test and chi-square.

Findings POST incidence was significantly higher in the $\mathrm{MCL}$ group compared to other two groups $(P<0.05)$. Duration of laryngoscopy was significantly longer in the MCL group compared to the GSL group (8.2 \pm 2.4 vs. $6.8 \pm 1.7 ; P=0.02)$. Regarding hemodynamic variables, only heart rate in the LMA group 3 minutes after intervention was significantly lower compared to other two groups $(\mathrm{P}=0.02)$.

Conclusion POST incidence was higher after using GSL and hemodynamic changes were less by using LMA. The preferred method for older people or cardiovascular patients is LMA. Further studies are needed to confirm these results.

\section{Extended Abstract}

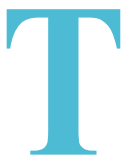

\section{Introduction}

racheal intubation is a conventional method to maintain an open airway in patients. This procedure is used in a variety of surgeries in the operating room. Various methods have been introduced for this purpose including using macintosh laryngoscope (MCL), glidescope laryngoscope (GSL), and laryngeal mask airway (LMA). Studies have shown that Postoperative Sore Throat (POST) is the most common complication of tracheal intubation; however, its actual incidence and extent are not well documented $[11,12]$. On the other hand,

\section{* Corresponding Author:}

Hamid Kayalha

Address: Department of Anesthesiology, Metabolic Disease Research Center, Qazvin University of Medical Sciences, Qazvin, Iran.

Tel: +98 (28) 33790620

E-Mail: hkayalha@qums.ac.ir 
researchers have presented controversial results regarding the superiority of each of these methods in terms of sympathetic reflexes (hypertension and tachycardia) $[13,14]$. So the best laryngoscope to achieve this goal is not clear. This study aimed to compare the POST incidence and hemodynamic changes after using MCL, GSL, and LMA.

\section{Materials and Methods}

This randomized double-blind clinical trial was performed on 90 patients aged 20-40 years undergoing elective surgery in Shahid Rajaee Hospital in Qazvin, Iran. Patients were randomly divided into three groups of MCL $(\mathrm{n}=30)$, GSL $(\mathrm{n}=30)$, and LMA $(\mathrm{n}=30)$. All interventions in the three groups were performed by an anesthesia resident with at least 30 successful intubation experiences in each of the three methods. For all patients, airway parameters (neck circumference and thyroid cartilage level) and demographic information (age, sex, height, and weight) were collected. Using a questionnaire, all patients were asked to report their sore throat 6-24 hours after surgery. Intubation problems were identified based on the Mallampati criterion. The duration of laryngoscopy and LMA placement were measured by a timer. In all patients, hemodynamic variables including heart rate and blood pressure (systolic, diastolic, overall) were recorded 1 minute before induction of anesthesia, and 3-5 minutes after intervention. To compare continuous variables, ANOVA and independent t-test, and to compare categorical variables, chi-square test were used in SPSS V. 22 software at a significance level of $\mathrm{P}<0.05$.

\section{Results}

There was no significant difference between the three study groups in terms of gender, age and body mass index and Mallampati criteria $(\mathrm{P}<0.05)$. Clinical and laryngoscopic information of patients in three groups are presented in Table 1. POST incidence was significantly higher in the MCL group compared to other two groups $(\mathrm{P}<0.05)$. Dura- tion of laryngoscopy in the two periods was significantly longer in the MCL group compared to the GSL group $(\mathrm{P}=0.02)$. Regarding hemodynamic variables, only heart rate in the LMA group 3 minutes after intervention was significantly lower compared to other two groups $(\mathrm{P}=0.02)$.

\section{Discussion}

The results of the present study showed that the incidence of POST in MCL group was higher compared to GSL and LMA groups. This is consistent with the results of other studies [15-17]. Aqil et al. [18] also showed that the incidence and severity of POST 6 hours after surgery in the MCL group was significantly higher than in the GSL group. Najafi et al. [19] also reported similar results. In this method, since direct vision is required, significant pressure is applied for inserting tube which can cause soft tissue damage and POST.

One of the factors that is effective in the occurrence of POST after anesthesia is the duration of intubation [12]. In the present study, no significant difference was observed in the duration of intubation between the three groups. The results of previous studies in this regard are contradictory. For example, Aqil et al. [18] reported that the duration of intubation in the MCL group was longer than in the GSL group, while Healy et al. [24] showed that its duration was longer in the GSL group. This difference may be due to the lack of hand-eye coordination or better vision for facilitating and accelerating intubation, both of which are dependent on the skill of the performer.

There was no significant difference between the groups in terms of hemodynamic variables such as heart rate, blood pressure (systolic, diastolic, overall); however, the heart rate was lower 3 minutes after anesthesia and intervention by the LMA method than by the other two methods. Woo et al. [25] also found no significant changes in systolic and diastolic blood pressure in both MCL and GSL groups;

Table 1. Clinical and laryngoscopic characteristics of patients in three groups

\begin{tabular}{|c|c|c|c|c|c|}
\hline Variables & & MCL & GSL & LMA* & $\mathbf{P}$ \\
\hline \multirow{2}{*}{ Postoperative Sore Throat (No. (\%)) } & $6-8 \mathrm{~h}$ after & $21(70)$ & $10(33.3)$ & $9(30)$ & $0.003^{* *}$ \\
\hline & $24 \mathrm{~h}$ after & $10(33.3)$ & $4(13.3)$ & $3(10)$ & $0.04 * *$ \\
\hline \multicolumn{2}{|l|}{ Duration of intubation (Mean \pm SD) MIN } & $12 \pm 1.9$ & $11.4 \pm 2$ & $10.9 \pm 2.5$ & 0.1 \\
\hline \multicolumn{2}{|l|}{ Duration of laryngoscopy (Mean \pm SD) SEC } & $8.2 \pm 2.4$ & $6.8 \pm 1.7$ & - & $0.02 * *$ \\
\hline \multirow{2}{*}{ Number of intubation attempts (No. (\%)) } & 1 & $22(75.9)$ & $25(83.3)$ & $22(73.3)$ & \multirow{2}{*}{0.6} \\
\hline & 2 & $7(24.1)$ & $5(16.7)$ & $8(26.7)$ & \\
\hline \multirow{2}{*}{\multicolumn{2}{|c|}{$\begin{array}{l}\text { Laryngeal Mask Airway } \\
* \text { Significant }(\mathrm{P}<0.05)\end{array}$}} & & \multirow{2}{*}{\multicolumn{3}{|c|}{$\begin{array}{l}\text { The Journal of } \\
\text { Qazvin University of Medical Sciences (JQUMS) }\end{array}$}} \\
\hline & & & & & \\
\hline
\end{tabular}


however, the heart rate of MCL used patients increased immediately after intubation. The discrepancy in results can be due to the different sample size, age of the patients and the type of used device.

Overall, it can be concluded that LMA is a preferred method in the elderly or people with cardiovascular disease. Further studies are needed to confirm these results. In future studies, it is recommended that the confirmation of POST results be performed using other methods and compared with one another to determine the effectiveness and complications of each method.

\section{Ethical Considerations}

\section{Compliance with ethical guidelines}

This is a registered clinical trial (Code: IRC T20180207038661N1) approved by the Research Ethics Committee of Qazvin University of Medical Sciences (Code: IR.QUMS.REC.1395.192). Informed consent was obtained from the all patients.

Funding

This study did not receive any specific grant from funding agencies in the public, commercial, or not for profit sectors.

\section{Authors' contributions}

Conceptualization, data collection and draft preparation: Mohammad Ali Masoumifar and Hamid Kayalha; Data analysis and interpretation: Mahdi Ebtehaj and Ali Akbar Shafikhani; Editing and review: All Authors.

\section{Conflicts of interest}

The authors declared no conflicts of interest. 


\title{
مقايسه بروز گَلودردو تغييرات هموديناميك در مديريت راه هوايى به وسيله لارنكَوسكوب مكينتاش، كَالايدوسكوب و ماسك حنجرواسي
}

\author{
محمدعلى معصومىفر'، مهدى ابتهاج' ه، "حميد كيالها' ه، علىاكبر شفيعخانى' (1)

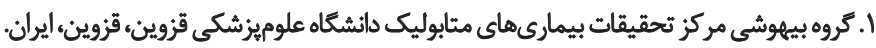

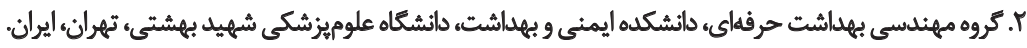

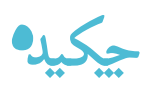

إنمينه مطالعات نشان دادهاند كه كلودرد يس از عمل، شايعترين عارضه الوله كذارى تراشه است، با اين حال بروز واقعى ودرجه آن بهخوبى

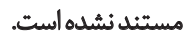

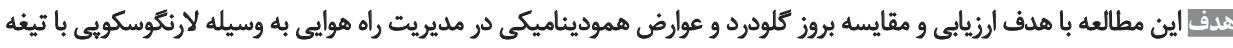

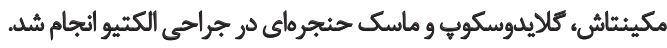

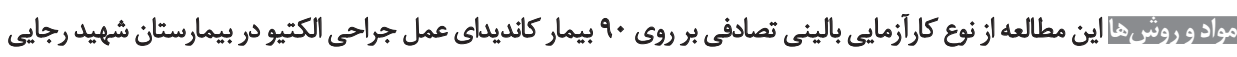

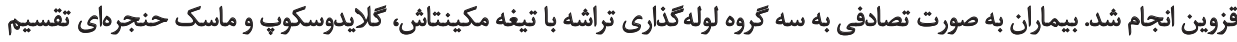

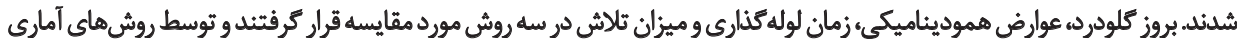

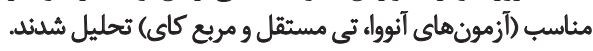

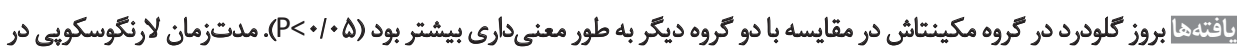

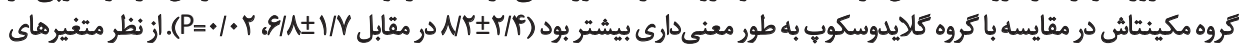

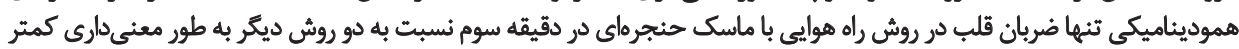
بود (P= (P)

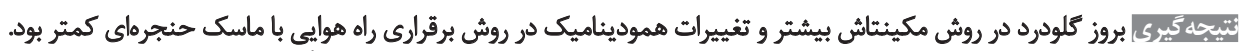

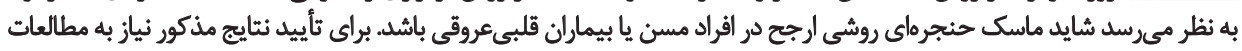
بيشترى در اين زميئه است.
\end{abstract}

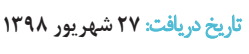

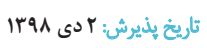
تاريخ انتشار: آبهمن

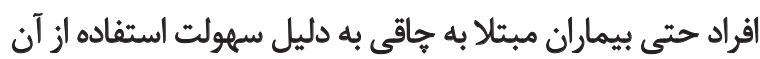
dale

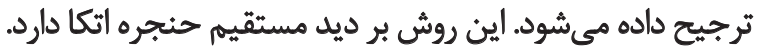

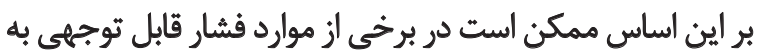

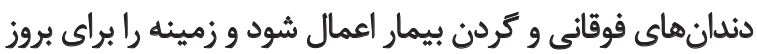

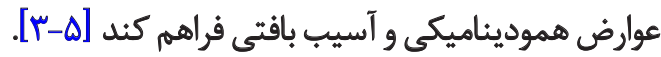
جاى كذارى LMA نيز يك وسيله نسبتاً جديد براي ساماندهي

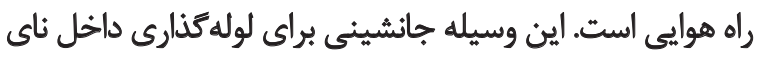

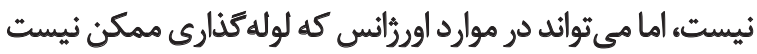

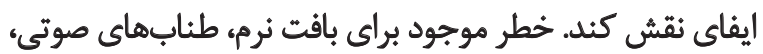

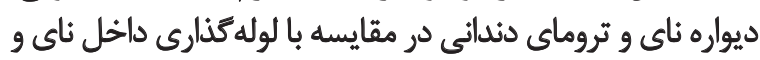

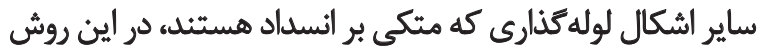
ㄷ..…….

\footnotetext{
* نويسئده مسئول: حميد كيالهيا نشانى: قزوين، دانشكاه علوميز شكيى قزوين، مركز تحقيقات بيماري هاي متابوليك، كروه بيهوشى.

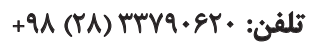
hkayalha@qums.ac.ir رايانامن:
} 
دستكاه تنفسى، آسيب دهان و حنجره و و ابتلا به ماكروكلوسيا

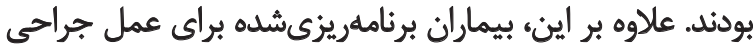

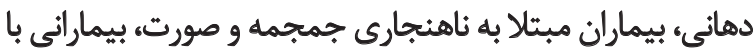

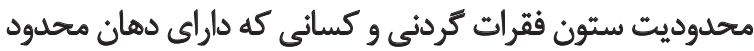

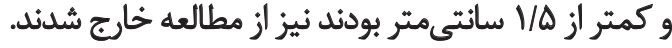
مانيتورينگ بيماران با استفاده از الكتروكارديوكرام، يالس

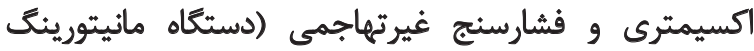

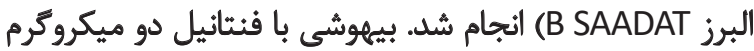

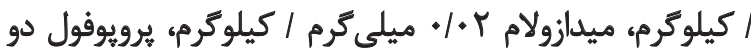

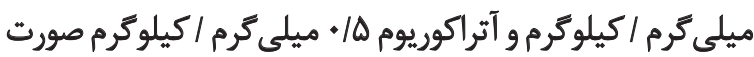

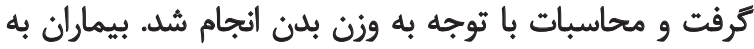

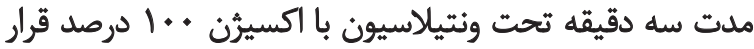

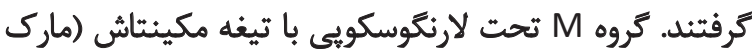

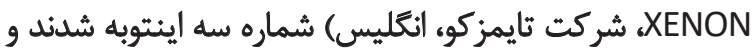

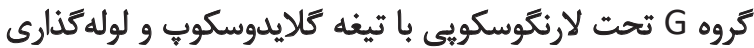

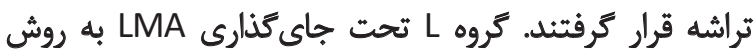

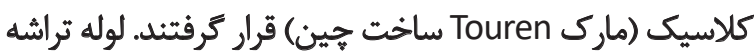

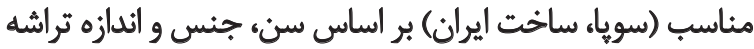

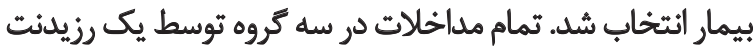

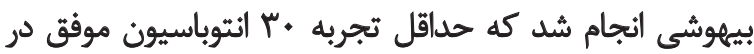

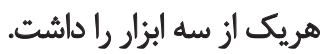

ارزيابىهاى كامل قبل از عمل براى تمام بيماران انجام شد.

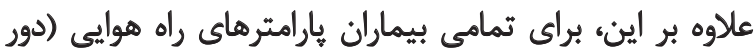

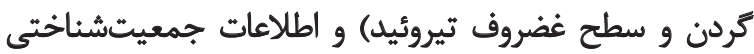

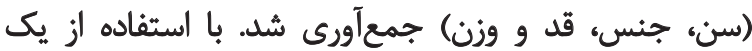

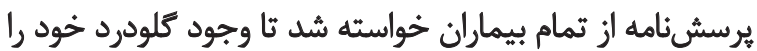

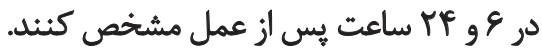

مشكلات لولهكذارى بر اساس معيار مالامياتى مشخص

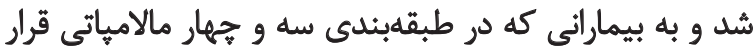

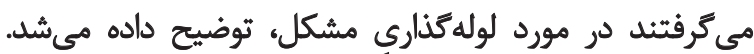

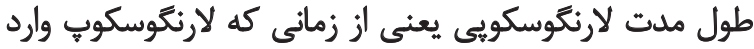

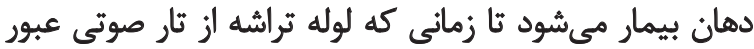

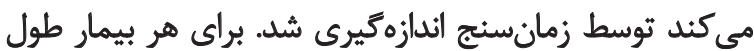

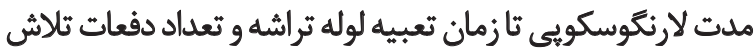

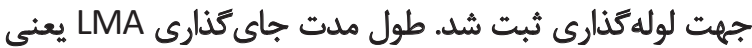

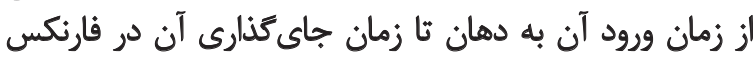

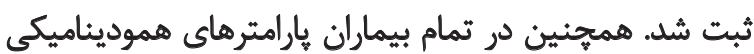

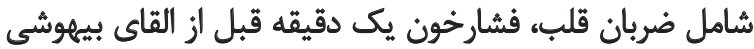

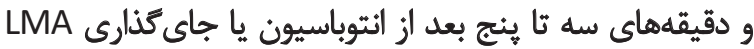
قيت شينل.

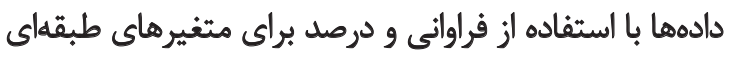
و ميانكين و انحراف معيار براى متغيرهاى خيوسته الرائه شدند.
به طور قابل ملاحظهاى كمتر است [ه-V]].

مطالعات نشان دادهاند لارنكوسكوب تصويرى (كلايدوسكوب)

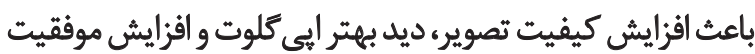

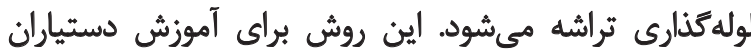

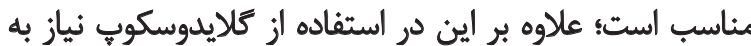

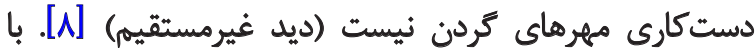

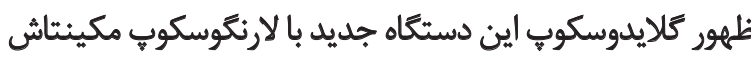

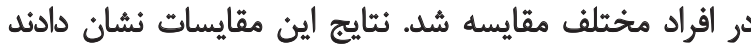

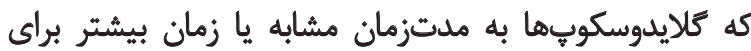

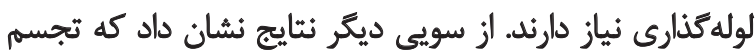

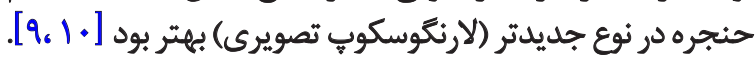
مطالعات نشان دادهاند كه كلودرد يس إز عمل، شايعترين

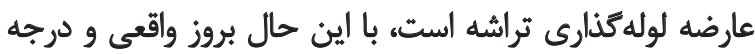

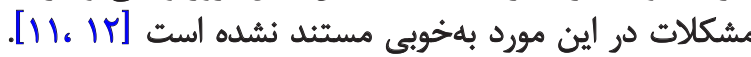

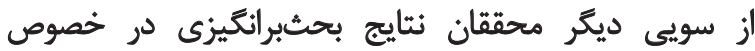

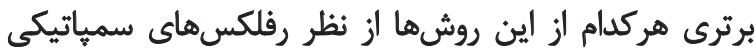

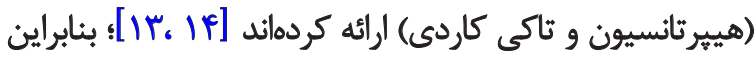

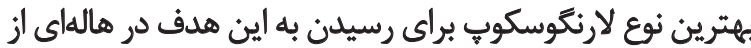

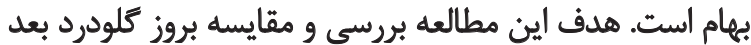

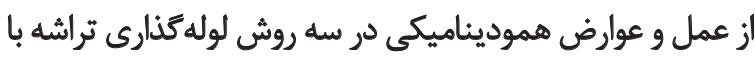

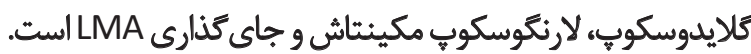

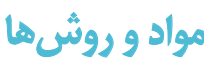
در اين مطالعه كار آزمايى بالينى تصادفى دوسوكور با كُد بندا

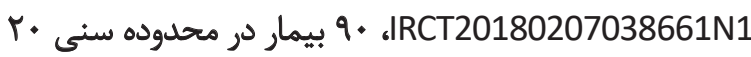

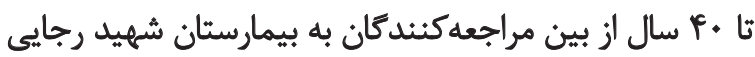

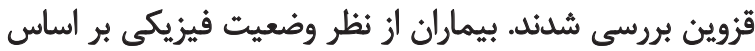

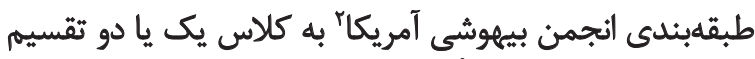

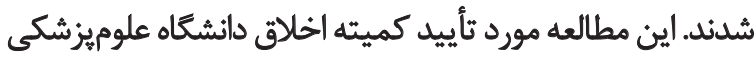

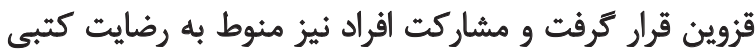

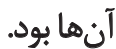

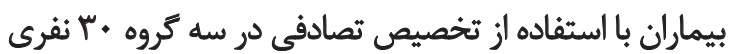

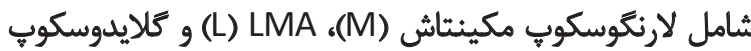

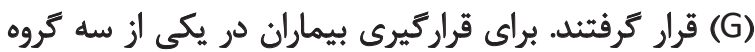

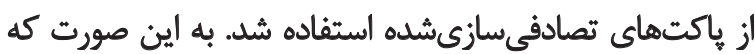

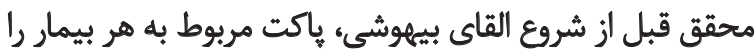

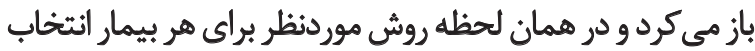

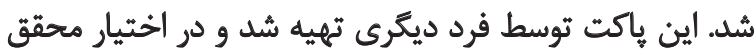

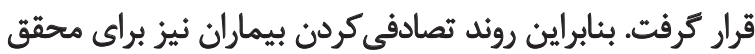

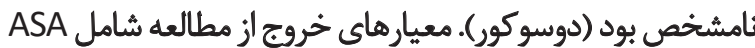

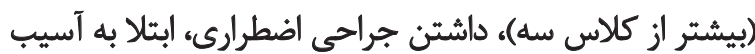

2. American society of anesthesiologists (ASA) 
يس از بيهوشى در روش LMA نسبت به دو روش ديكر كمتر بود

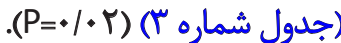

\section{بحث ونتيجنانئيرى}

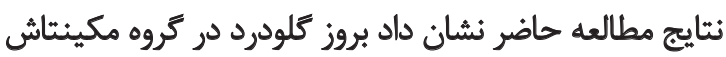

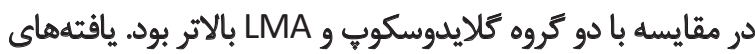

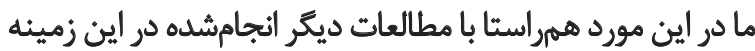

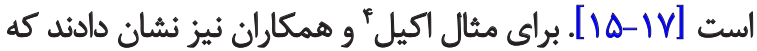

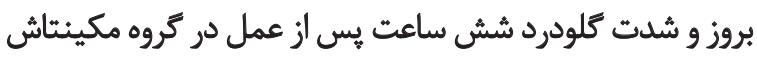

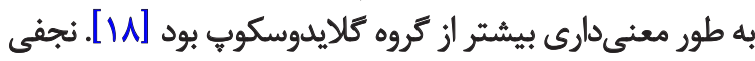

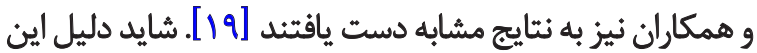

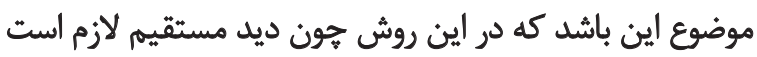

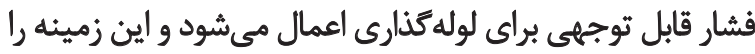

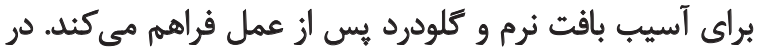

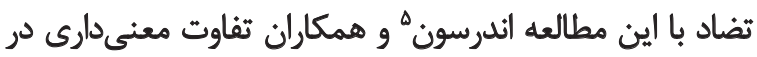

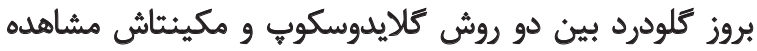

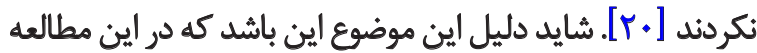

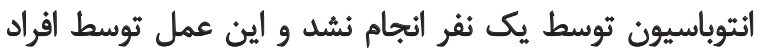

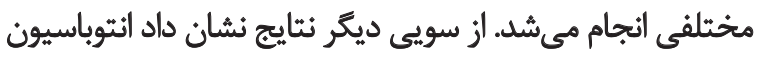

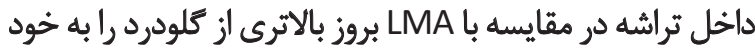

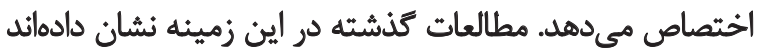

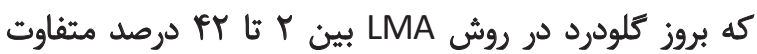

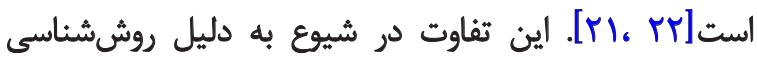

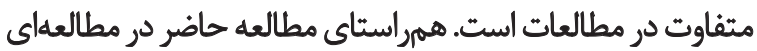

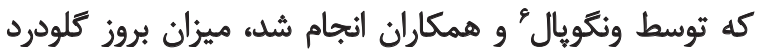

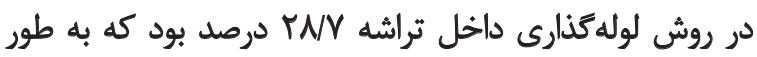

4. AQIL

5. Andersen

6. Venugopal

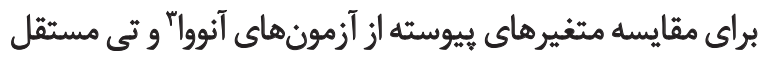

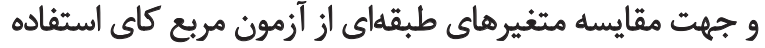

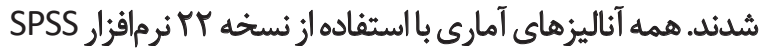

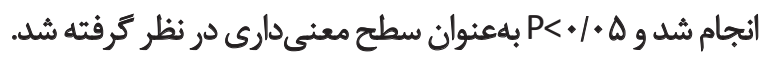

يا

از نظر جنسيت، سن و شاخص توده بدنى و معيار مالامياتى

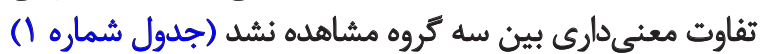

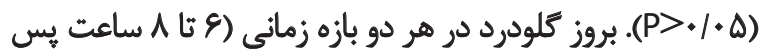

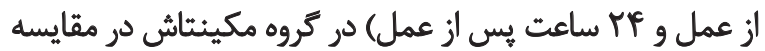

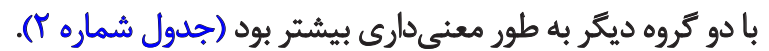
طول مدت انتوباسيون به ترتيب در كروه مكينتاش،

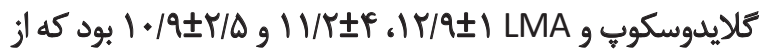

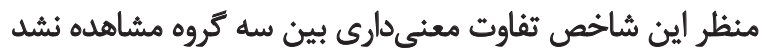

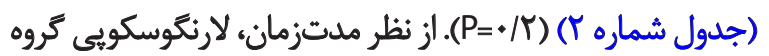

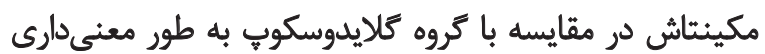

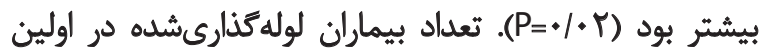

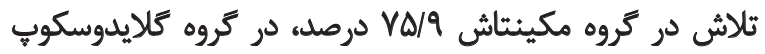

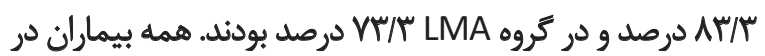

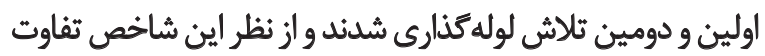

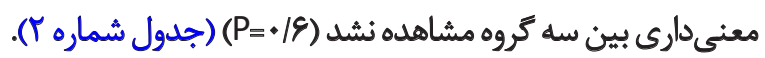

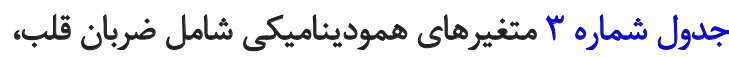

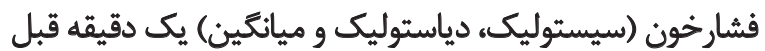

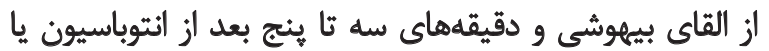

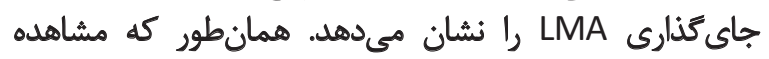
مىشود در ميان اين شاخصها تنها ضربان فلب در سه دقيقه

3. ANOVA

جدول (. مقايسه ويرُكى جمعيتشناختى و لوله كذارى بيماران در سه كروه موردمطالعه (هر كروه •ب نفر)

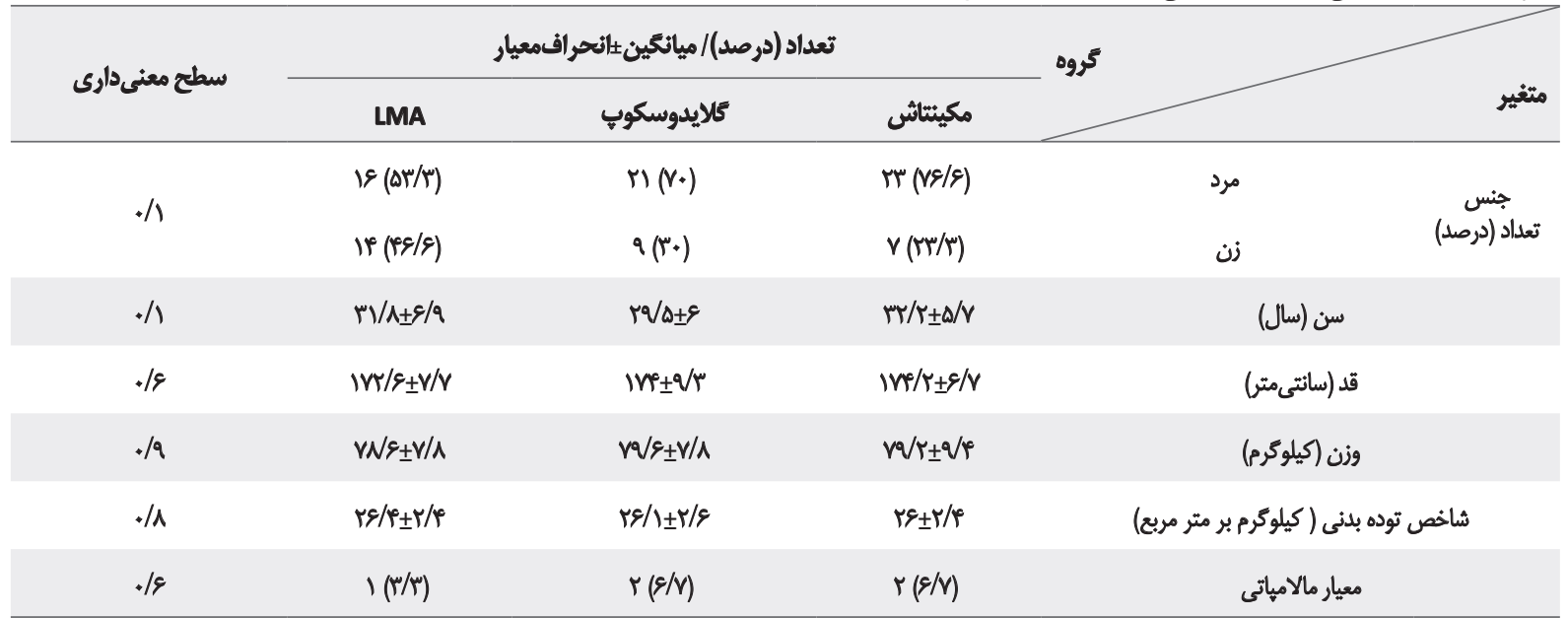

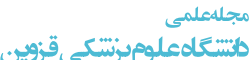


جدول †. مقايسه اطلاعات بالينى و لارئكوسكويى بيماران در سه كروه موردمطالعه

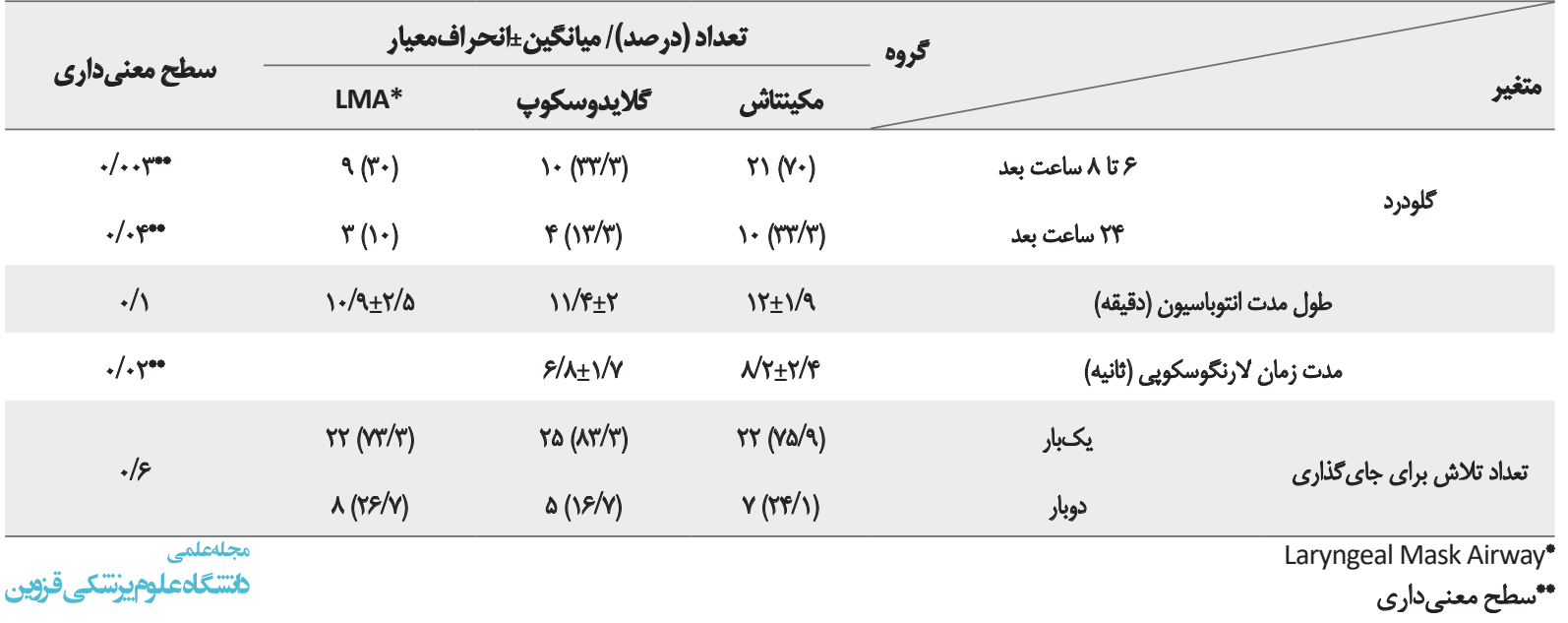

جدول "ا. مقايسه متغيرهاى هموديثاميكى بيماران در سه كروه موردمطالعه

\begin{tabular}{|c|c|c|c|c|c|}
\hline \multirow{2}{*}{ سطح معني 10 ري } & \multicolumn{3}{|c|}{ ميانكين+انحراف معيار } & \multirow{2}{*}{ زمان } & \multirow{2}{*}{ متغير } \\
\hline & LMA & كملايدوسكوب & مكينتاش & & \\
\hline.$/ p$ & $I Y \Delta \pm I V / Y$ & $|r| \pm \mid Q / \Lambda$ & $1 r \cdot \pm 11 / 4$ & قبل از جاى ثنارى & \multirow{3}{*}{ 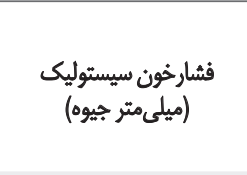 } \\
\hline$\cdot / r$ & $1.9 \pm 19 / 8$ & $\| \varepsilon \pm \pi r / F$ & $\| V+\mid \alpha / 8$ & دقيقه ب & \\
\hline.$/ \%$ & $110 \pm 12 / 1$ & $\| \Delta \pm|N|$ & $11 \cdot \pm 1 \% / 9$ & دقيقه ه & \\
\hline.$/ 8$ & $r \pm r 1$ & $s q_{ \pm} \mid f / \lambda$ & $n \pm \pm 1 r / \Delta$ & قبل از جاي كذارى & \multirow{3}{*}{ 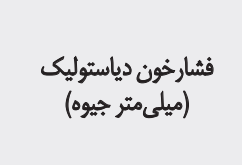 } \\
\hline.$/ 9$ & $V \cdot \pm I f / 8$ & $v+ \pm 1 r / 9$ & $9 q \pm 1 r / \Delta$ & دقيقه ب & \\
\hline.$/ \Delta T$ & $n_{ \pm} \pm 1 r / q$ & $\Delta r \pm 1 Q / r$ & $v e \pm 1 r / s$ & دقيقه ه & \\
\hline.$/$ & $91 \pm 1 \cdot / 1$ & $M \pm 1 \theta / \Lambda$ & $\vartheta \pm I V / A$ & قبل از جاى كذارى & \multirow{3}{*}{ فشارخون ميائكين (ميلى متر } \\
\hline.$/ 8$ & $19 \pm r r / \Delta$ & $\Lambda \Delta \pm 1 \% / \Delta$ & $A Y \pm i r / V$ & ل دقيقه ب & \\
\hline.$/ 9$ & $\Lambda \Delta \pm W A$ & $\Lambda \Delta \pm 1 \Delta / \Delta$ & $N \Delta \pm I r / V$ & دقيقه هـ & \\
\hline.$/ 4$ & $\Delta r \pm 11 / \Lambda$ & $\Delta_{i} \pm I V / r$ & $A Y \pm 1 Y / \Delta$ & قبل از جاى كذارى & \multirow{3}{*}{ ضربان قلب (ميلىمتر جيوه) } \\
\hline.$/ . r^{*}$ & $V A \pm 11 / V$ & $\Delta a \pm I r$ & $\Delta Y \pm 1 f / \Delta$ & دقيقه ب & \\
\hline$+/ r$ & $W \pm 11 / 8$ & $\Lambda T \pm I Y / r$ & $1 \cdot \pm 1 r / q$ & دقيقه هـ & \\
\hline
\end{tabular}

روش لوله كذارى مي تواند به علت مهارت فرد در اين مطالعه باشد.

يكى ديگ از عواملى كه در بروز درد كلو يس از بيهوشى مؤثر

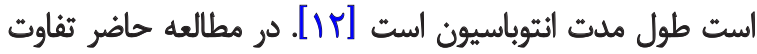

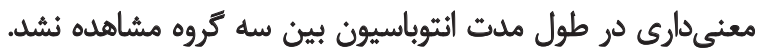

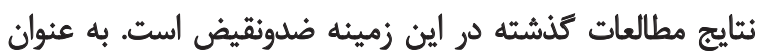

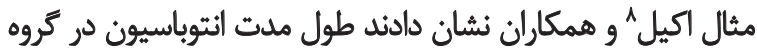

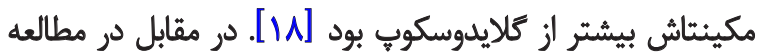

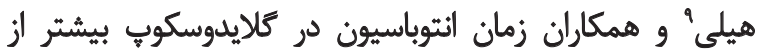

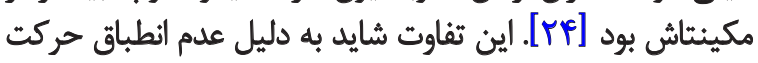

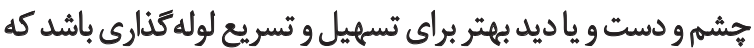

معنى دارى از روش LMA بيشتر بود [TY]. در مطالعه حاضر تعداد دفعات لولهَّارى بين سه روش تفاوت

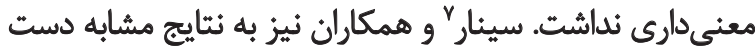

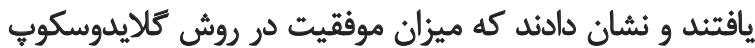

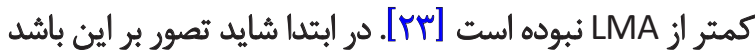

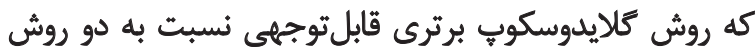

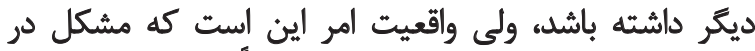

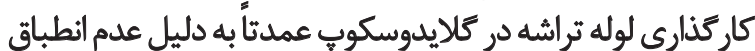

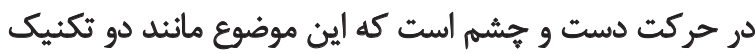

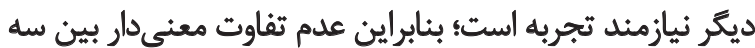




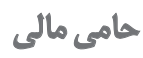

اين مطالعه هيج كمك مالى خاصى از سازمان هاي تأمين مالى

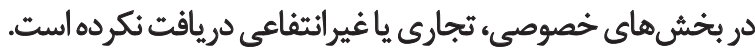

$$
\text { مشاركت نويسئد مكان }
$$

طراحى مطالعه، جمعآورى دادها و و تهييه بيشيشنويس:

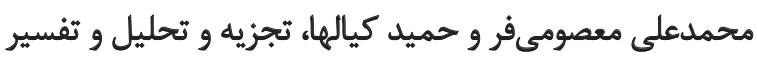

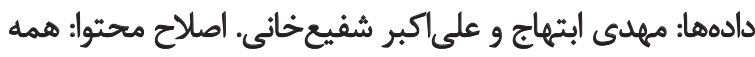

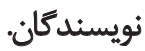

$$
\text { ت تعارض مثافع }
$$

بنابر اظهار نويسندكان اين مقاله هيج تعارض منافعى نداشته است.
هردوى اين عوامل در كنار مهارت، تابع فرد اجراكنيده است.

از نظر متغيرهاى هموديناميكى فشار خون سيستوليك،

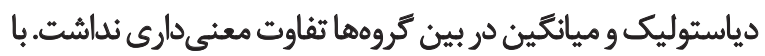

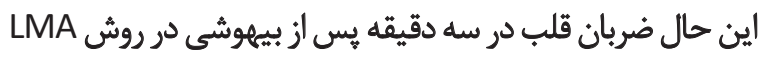

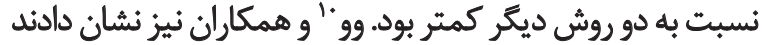

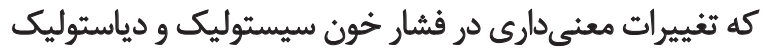

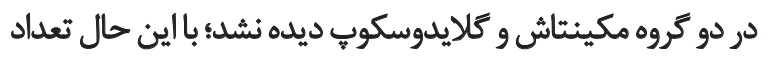

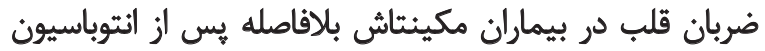

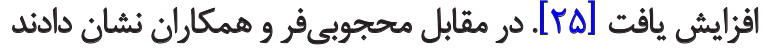

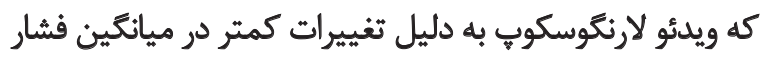

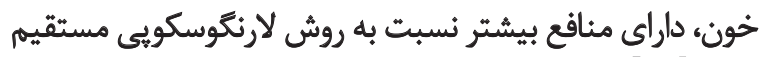

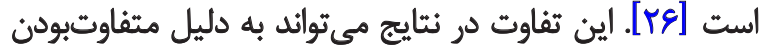
حجم نمونه، سن بيماران و نوع وسيله مورداستفاده باشد.

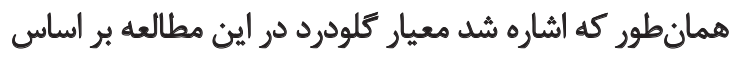

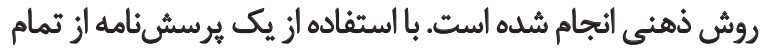

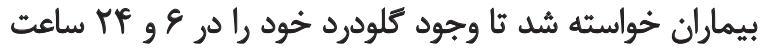

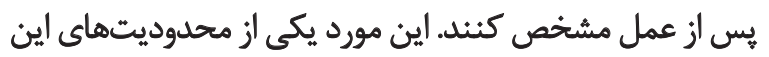

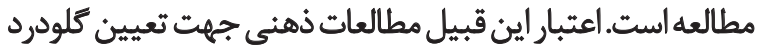

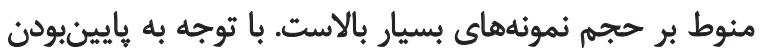

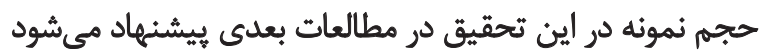

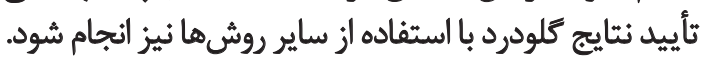

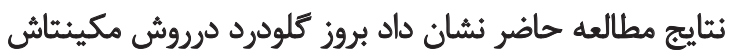

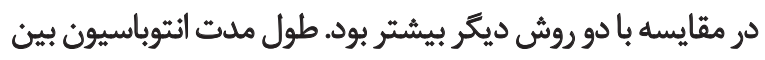

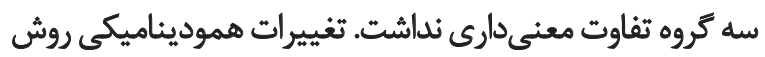

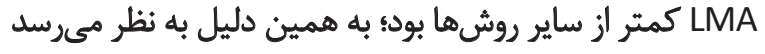

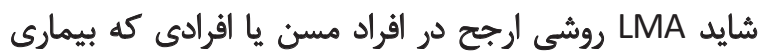

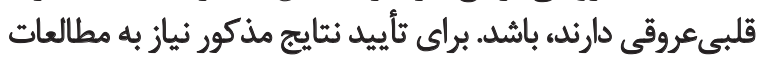

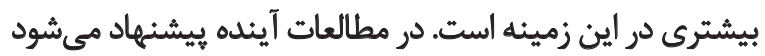

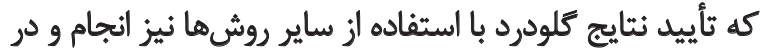

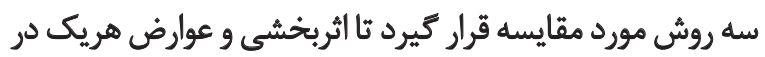
مقايسه با ديغرى مشخص شود مقائه فرار ميرد

مالامظات الخاقي

$$
\text { بيروى از اصول اخلاق ثئوهش }
$$

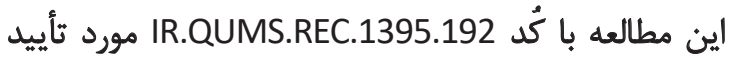

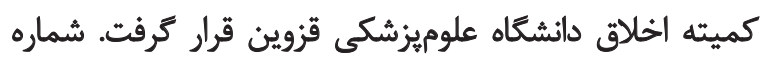

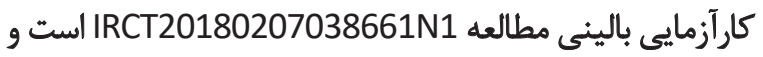
جهت انتشار مقاله از بيماران رضايت آكاهانه كرفته شد. 


\section{References}

[1] Higgs A, McGrath BA, Goddard C, Rangasami J, Suntharalingam $G$, Gale R, et al. Guidelines for the management of tracheal intubation in critically ill adults. Br J Anaesth. 2018; 120(2):32352. [DOI:10.1016/j.bja.2017.10.021] [PMID]

[2] Meissen $\mathrm{H}$, Johnson L. Managing the airway in acute care patients. Nurse Pract. 2018; 43(7):23-9. [DOI:10.1097/01. NPR.0000534937.35090.f1] [PMID]

[3] Nasim F, Chae J, Goel S. Endotracheal intubation in critically III patients: Direct laryngoscopy, complications, and cardiac arrest. Am J Respir Crit Care Med. 2018; 197(12):1625-7. [DOI:10.1164/rccm.201709-1884RR] [PMID]

[4] Aggarwal H, Kaur S, Baghla N, Kaur S. Hemodynamic response to orotracheal intubation: Comparison between Macintosh, McCoy, and C-MAC video laryngoscope. Anesth Essays Res. 2019; 13(2):308-12. [DOI:10.4103/aer.AER_7_19] [PMID] [PMCID]

[5] Tosi F, Genovese O, Jovanovic T, Visocchi M. Management of anaesthesia. In: Visocchi M, editor. New Trends in Craniovertebral Junction Surgery, Acta Neurochirurgica Supplement. Vol. 125. Cham: Springer; 2019. p. 381-6. [DOI:10.1007/978-3-31962515-7_54] [PMID]

[6] Qureshi MJ, Kumar M. Laryngeal mask airway versus bagmask ventilation or endotracheal intubation for neonatal resuscitation. Cochrane Database Syst Rev. 2018; 3:CD003314. [DOI:10.1002/14651858.CD003314.pub3] [PMCID]

[7] Roberts KD, Brown R, Lampland AL, Leone TA, Rudser KD, Finer $N N$, et al. Laryngeal mask airway for surfactant administration in neonates: A randomized, controlled trial. J Pediatr. 2018; 193:40-6.e1. [DOI:10.1016/j.jpeds.2017.09.068] [PMID]

[8] Hurley RW, Murphy J, Wu C. Acute Postoperative Pain. U: Miller RD, ur. Miller's Anesthesia. 7th ed. New York: Philadelphia: ELSEVIER SAUNDERS; 2015. 1533-56.

[9] Zhang B, Gurnaney HG, Stricker PA, Galvez JA, Isserman RS, Fiadjoe JE. A prospective observational study of technical difficulty with glidescope-guided tracheal intubation in children. Anesth Analg. 2018; 127(2):467-71. [DOI:10.1213/ ANE.0000000000003412] [PMID]

[10] Nandakumar KP, Bhalla AP, Pandey RK, Baidya DK, Subramaniam R, Kashyap L. Comparison of Macintosh, McCoy, and Glidescope video laryngoscope for intubation in morbidly obese patients: Randomized controlled trial. Saudi J Anaesth. 2018; 12(3):433-9. [DOI:10.4103/sja.SJA_754_17] [PMID] [PMCID]

[11] Safaeian R, Hassani V, Movasaghi GR, Alimian M, Faiz HR. Postoperative respiratory complications of laryngeal mask airway and tracheal tube in ear, nose and throat operations. Anesth Pain Med. 2015; 5(4):e25111. [DOI:10.5812/ aapm.25111] [PMID] [PMCID]

[12] Lee JY, Sim WS, Kim ES, Lee SM, Kim DK, Na YR, et al. Incidence and risk factors of postoperative sore throat after endotracheal intubation in Korean patients. J Int Med Res. 2017; 45(2):744-52. [DOI:10.1177/0300060516687227] [PMID] [PMCID]
[13] Sener EB, Ustun E, Ustun B, Sarihasan B. Hemodynamic responses and upper airway morbidity following tracheal intubation in patients with hypertension: Conventional laryngoscopy versus an intubating laryngeal mask airway. Clinics (Sao Paulo). 2012; 67(1):49-54. [DOI:10.6061/clinics/2012(01)08] [PMID] [PMCID]

[14] Hashemian AM, Zamani Moghadam Dolooa H, Saadatfar M, Moallem R, Moradifar M, Faramarzi R, et al. Effects of intravenous administration of fentanyl and lidocaine on hemodynamic responses following endotracheal intubation. Am J Emerg Med. 2018; 36(2):197-201. [DOI:10.1016/j.ajem.2017.07.069] [PMID]

[15] Puchner W, Drabauer L, Kern K, Mayer C, Bierbaumer J, Rehak $\mathrm{PH}$, et al. Indirect versus direct laryngoscopy for routine nasotracheal intubation. J Clin Anesth. 2011; 23(4):280-5. [DOI:10.1016/j.jclinane.2010.10.003] [PMID]

[16] Teoh WHL, Shah MK, Sia ATH. Randomised comparison of Pentax AirwayScope and Glidescope for tracheal intubation in patients with normal airway anatomy. Anaesthesia. 2009; 64(10):1125-9. [DOI:10.1111/j.1365-2044.2009.06032.x] [PMID]

[17] Jones PM, Armstrong KP, Armstrong PM, Cherry RA, Harle CC, Hoogstra J, et al. A comparison of glidescope videolaryngoscopy to direct laryngoscopy for nasotracheal intubation. Anesth Analg. 2008; 107(1):144-8. [DOI:10.1213/ ane.0b013e31816d15c9] [PMID]

[18] Aqil M, Khan MU, Mansoor S, Mansoor S, Khokhar RS, Narejo AS. Incidence and severity of postoperative sore throat: A randomized comparison of Glidescope with Macintosh laryngoscope. BMC Anesthesiol. 2017; 17:127. [DOI:10.1186/s12871017-0421-4] [PMID] [PMCID]

[19] Najafi A, Imani F, Makarem J, Khajavi MR, Etezadi F, Habibi Sh, et al. Postoperative sore throat after laryngoscopy with macintosh or glide scope video laryngoscope blade in normal airway patients. Anesth Pain Med. 2014; 4(1):e15136. [DOI:10.5812/ aapm.15136] [PMID] [PMCID]

[20] Andersen LH, Rovsing ML, Olsen KS. GlideScope videolaryngoscope vs. Macintosh direct laryngoscope for intubation of morbidly obese patients: A randomized trial. Acta Anaesthesiol Scand. 2011; 55(9):1090-7. [DOI:10.1111/j.13996576.2011.02498.x] [PMID]

[21] Bernstein M, Moniodis A, Fishman E, Krinsley J. Laryngeal mask airway use during bronchoscopic flexible cryoprobe use. Chest. 2018; 154(4 Suppl):846A. [DOI:10.1016/j. chest.2018.08.768]

[22] Venugopal A, Jacob RM, Koshy RC. A randomized control study comparing the pharyngolaryngeal morbidity of laryngeal mask airway versus endotracheal tube. Anesth Essays Res. 2016; 10(2):189-94. [DOI:10.4103/0259-1162.174466] [PMID] [PMCID]

[23] Cinar O, Cevik E, Yıldırım AO, Yasar M, Kilic E, Comert B. Comparison of GlideScope video laryngoscope and intubating laryngeal mask airway with direct laryngoscopy for endotracheal intubation. Eur J Emerg Med. 2011; 18(2):117-20. [DOI:10.1097/MEJ.0b013e32833e79e6] [PMID] 
[24] Healy DW, Picton P, Morris M, Turner C. Comparison of the glidescope, CMAC, storz DCl with the Macintosh laryngoscope during simulated difficult laryngoscopy: A manikin study. BMC Anesthesiol. 2012; 12:11. [DOI:10.1186/1471-2253-12-11] [PMID] [PMCID]

[25] Woo CH, Kim SH, Park JY, Bae JY, Kwak IS, Mun SH, et al. Macintosh laryngoscope vs. Pentax-AWS video laryngoscope: Comparison of efficacy and cardiovascular responses to tracheal intubation in major burn patients. Korean J Anesthesiol. 2012; 62(2):119-24. [DOI:10.4097/kjae.2012.62.2.119] [PMID] [PMCID]

[26] Mahjoubifar M, Borjian Boroojeny Sh. Hemodynamic changes during orotracheal intubation with the glidescope and direct laryngoscope. Iran Red Crescent Med J. 2010; 12(4):406-8. http://ircmj.com/articles/77390.html 